\section{EMBRYRIDDLE \\ Aeronautical University}

SCHOLARLY COMMONS
International Journal of Aviation, Aeronautics, and Aerospace

\title{
Small Unmanned Aircraft System Operator Compliance with Visual Line of Sight Requirements
}

\author{
Ryan J. Wallace \\ Embry-Riddle Aeronautical University, ryan.wallace@erau.edu \\ Kristine M. Kiernan \\ Embry-Riddle Aeronautical University, kiern4fd@erau.edu \\ John Robbins \\ Embry-Riddle Aeronautical University, robbinsj@erau.edu \\ Tom Haritos \\ Kansas State University, Applied Aviation Research Center, tharitos@k-state.edu
}

Follow this and additional works at: https://commons.erau.edu/ijaaa

Part of the Aviation Safety and Security Commons

\section{Scholarly Commons Citation \\ Wallace, R. J., Kiernan, K. M., Robbins, J., \& Haritos, T. (2019). Small Unmanned Aircraft System Operator Compliance with Visual Line of Sight Requirements. International Journal of Aviation, Aeronautics, and Aerospace, 6(2). https://doi.org/10.15394/ijaaa.2019.1327}

This Article is brought to you for free and open access by the Journals at Scholarly Commons. It has been accepted for inclusion in International Journal of Aviation, Aeronautics, and Aerospace by an authorized administrator of Scholarly Commons. For more information, please contact commons@erau.edu. 
On September 21, 2017, a small unmanned aircraft systems (sUAS) operator launched his DJI Phantom 4 from the Dyker Beach Park shoreline on an evening recreational flight over the Hudson River estuary (National Transportation Safety Board [NTSB], 2017). Flying perpendicular to the shoreline, the sUAS operator piloted the aerial vehicle more than 2.5 miles away, "well beyond visual line of sight (BVLOS)" (NTSB, 2017, p. 1). The investigation revealed the operator was flying solely using reference to the user interface map rather than in combination with visual contact with the vehicle, and was unaware of the proximity of a military UH-60M helicopter conducting a low altitude orientation flight in the same area. At 7:20pm, the helicopter reported seeing the sUAS rapidly closing on his position. Despite attempting to perform an evasive vertical maneuver, the helicopter struck the small unmanned aircraft, causing damage to one of the UH60 's rotor blades (NTSB, 2017). Forensic records collected from the sUAS flight data logs indicated this was not the first instance in which the operator flew his craft beyond visual line of sight. On a second flight earlier that evening, the operator flew up to 1.8 miles away, which the NTSB stated was "unlikely to be within visual line of sight" (NTSB, 2018, p. 1).

\section{Problem}

The potential hazards associated with BVLOS flight represent a clear danger to manned aircraft operators and other National Airspace System (NAS) users. Currently, no data exists to accurately assess the distance at which sUAS operators are flying their aerial vehicles. The authors sought to determine the extent of sUAS flights conducted beyond visual line of sight without appropriate waivers or risk mitigation.

\section{Purpose}

The purpose of this study was to assess sUAS operator practices with an emphasis on the range and visibility characteristics between the operator and aerial vehicle. This data will be used to establish a baseline of UAS operator flight behavior as well as generate UAS policy and safety recommendations.

\section{Research Questions}

1. How far away do sUAS operators typically fly their unmanned aircraft?

2. What proportion of sampled flights were conducted Beyond Visual Line of Sight (BVLOS)? 


\section{Background}

\section{Existing Regulations}

Federal Aviation Administration rules require commercial sUAS operations to be carried out so that the remote pilot, visual observer, and person manipulating the flight controls can see the unmanned aircraft throughout the entire flight (14 CFR 107.31, 2016). Similarly, the FAA Modernization and Reform Act (2012) requires individuals operating unmanned aircraft under the Special Rule for Model Aircraft to fly within visual line of sight. Existing regulatory guidance, however, does not codify measurable criteria to determine compliance with these visual line of sight rules.

In responding to public comments to NPRM for Part 107, the FAA clarified its position and rationale for the rule's visual line of sight provisions. Interestingly, the FAA declined to set a definable limit for determining what distance constitutes visual line of sight. According to the FAA (2016):

A prescriptive numerical limit would not take into account situationaldependent operating factors and may preclude operations that could otherwise be conducted safely. Additionally, no commenter provided data to substantiate the belief that a numerical standard would provide a higher level of safety than the visual line of sight standard proposed in the NPRM. (p. 132-133)

Yet, when commenting about Part 107's provisions for transporting property for compensation, the FAA did indicate an approximation for visual line of sight criteria, "the visual line of sight restriction limits the area of operation to a circle with only about 1-mile radius around the remote pilot in command, depending on the visibility conditions at the time of the operation" (FAA, 2016, p. 50). The lack of a clearly-defined visual line of sight standard is likely to make both compliance and enforcement of the visual line of sight provisions problematic.

Nevertheless, the agency has issued only a small number of waivers for BVLOS flight. As of December 21, 2018, the FAA had only authorized 29 entities authority to exceed 14 CFR Part 107.31 visual line of sight requirements (FAA, n.d., 2018).

\section{Hazards of BVLOS Flight}

Flights beyond visual line of sight have the potential to be particularly hazardous, since they limit the situational awareness of operators. Known as the 
soda straw effect, the reduced field of view of visual information can diminish hazard recognition, and ultimately decrease operational situational awareness (Terwilliger, 2012). As a result, flight beyond visual line of sight requires operators to determine how to assess the UAS's location and trajectory, track cooperative and non-cooperative aircraft, observe other surrounding hazards, and ensure UAS connectivity (PrecisionHawk, 2018). According to the FAA's 14 CFR Part 107 Waiver Safety Explanation Guidelines, UAS operators who apply for 14 CFR Part 107.31 waivers to fly beyond visual line of sight must address the following issues (FAA, n.d.):

- Obtaining continuous position, altitude, attitude, and movement updates from the unmanned aircraft

- Ensure the UAS remains within the designated area of operation

- Avoidance of aircraft, flight over people, ground structures and other obstacles

- Ensure the unmanned aircraft can be visibly seen by manned aircraft at a distance of at least $3 \mathrm{SM}$

- Method to ensure remote pilot is alerted of degraded unmanned aircraft functionality

- Plan for ensuring operation participants remain aware of the UAS operational status

- Ensure UAS compliance with weather restrictions

- Fidelity of command, control, and communication systems

\section{Literature Review}

\section{Previous Research}

Several researchers have assessed the challenges associated with seeing unmanned aircraft. In a series of four experiments, Crognale (2009) evaluated the effectiveness of visual observer's acquisition of a Scan Eagle, fixed wing UAS with a 10.2-foot wingspan. Crognale reported a mean detection distance of 898 meters $(\sim 2,946$ feet $)$ for vehicles flying towards the observer, and 1,276 meters $(\sim 4,186$ feet) for vehicles flying away from the observer. In another study, Dolgov (2016) evaluated visual observer performance in daytime, dusk, and nighttime settings using small fixed-wing RQ-11B (4.5-foot wingspan) and Wasp III (2.4-foot wingspan) aircraft. Dolgov's study determined that during daytime conditions, the sUAS craft were visually acquired by visual observers at a mean distance of 0.72 $\mathrm{km}(2,362$ feet) for the RQ-11B and $0.76 \mathrm{~km}(2,493$ feet) for the Wasp III. In a recent in-flight experiment, Loffi, Wallace, Jacob, and Dunlap (2016) assessed that pilots using see-and-avoid procedures reliably detected small, quadcopter unmanned aircraft at ranges less than .10 SM ( 528 feet). 
It is notable that the resulting detection distances of the presented studies varied widely. According to Williams and Gildea (2014), other factors that potentially impact visibility of unmanned aircraft include: visual angle, obstructions, visual acuity, visual accommodation (focus), contrast, background, search time, and apparent motion. Crognale (2009) suggested that position uncertainty may also influence mean detection distances.

\section{Theoretical Visual Modeling}

While the previous field experiments provide a basis for practical spotting and tracking of unmanned aircraft, it is also necessary to evaluate the theoretical foundations of sight and object recognition. As one might expect, visual detection is directly affected by the relative size of the object being observed, measured in arc minutes. An arc minute is a unit of angular measurement equivalent to 1/60 of a degree.

In interpreting probability of visual detection curves originally produced by Greening (1976), Woo (2017, p. 46) makes the following observations:

a) Targets with visual angles less than one arc minute are unlikely to be seen

b) Targets with visual angles of at least 1 arc minute may be seen by those with normal (20/20) vision

c) Targets with visual angles greater than 10 arc minutes are likely to detected (but not necessarily recognized)

d) Targets become recognizable between $30 \%-40 \%$ of the time when they render a visual angle of 15 arc minutes or more

e) In four of the six models, targets become recognizable $50 \%$ to $100 \%$ of the time when the visual angle exceeds 30 arc minutes

According to Woo (2017), literature consistently applies a standard of 1.0 arc minutes as the minimum size of a target that a person with 20/20 normal visual acuity should be capable of seeing. The National Bureau of Standards uses this as the minimum resolution needed for readable signage (Howett, 1983). In addition to relative size, the visual acuity of the observer also affects object detection. Table 1 depicts the relationship between visual acuity and the critical visual angle necessary to achieve visual detection. 
Table 1

Snelling Notation Relationship to Visual Arc Size in Minutes

\begin{tabular}{llll}
\hline Snelling Notation & $\begin{array}{l}\text { Critical } \\
\text { Angle (minutes) }\end{array}$ & $\begin{array}{l}\text { Visual } \\
\text { population } \\
\text { (uncorrected) }\end{array}$ & $\begin{array}{l}\text { of } \\
\text { population } \\
\text { (corrected) }\end{array}$ \\
\hline $20 / 10$ & 0.5 & 1.1 & 1.5 \\
$20 / 15$ & 0.75 & 30.3 & 40.0 \\
$20 / 20$ & 1.0 & 53.9 & 72.9 \\
$20 / 30$ & 1.5 & 69.3 & 90.6 \\
$20 / 40$ & 2.0 & 75.8 & 95.1 \\
\hline
\end{tabular}

Depiction of the relationship between visual acuity in Snelling notation, the minimum number of arc minutes that can be seen with each Snelling rating and subsequent percentage of population who have such visual acuity or better - both uncorrected and with correction. Adapted from "Visual Detection of Small Unmanned Aircraft: Modeling the Limits of Human Pilots," by G. S. Woo, 2017, https://commons.erau.edu/edt/350, p. 32. Used with permission.

This visual modeling is important, as it can be used as a basis for determining if it is possible for unmanned aircraft or other objects to be visually detected by remote pilots or visual observers.

\section{Methodology}

The authors employed an applied, exploratory research approach for this study. A DJI AeroScope was deployed near an urban airport for 30 days to detect sUAS activity. The AeroScope is a device that detects RF command and control datalink signals between DJI remote controllers and their aerial vehicles. The authors extracted telemetry plots of detected sUAS controllers and unmanned aircraft within the sample area. Lateral distance information was calculated from the collected GPS coordinates using an Excel-based geolocation algorithm. Lateral distance data was converted to slant range using a trigonometric calculation. A maximum value algorithm was used to determine the maximum distance flown from the operator during each detected sUAS flight.

The maximum flight distance values were used to determine operator visibility of each aerial vehicle. The sUAS model information was extracted from the telemetry data and a size value assigned to each detection based on the largest diagonal cross-section of the aerial vehicle. The authors obtained relative diagonal model size information from published manufacturer specifications. This represents the maximum possible visual size of the vehicle. While the aerial vehicle would normally be seen from the side rather than from directly above or below, the 
authors elected to use diagonal size to determine the best possible visual condition. This selection should improve study validity by ensuring that unmanned aircraft assessed to be beyond visual line of sight were truly impossible to see. Size information was used to reverse-calculate maximum visual arc detection distances for each UAS model, based on Greening's (1976) visual detection modeling. The reverse calculation yielded five visibility categories: less than 1 arc-minute, 1 arcminute, 10 arc-minutes, 15 arc-minutes, and 30 arc-minutes. Maximum visibility distances for each UAS based on this modeling criteria are presented in Table 2. Maximum flight distance values were compared against the Greening model values to determine visibility characteristics. 
Table 2

Unmanned Aircraft Visual Distances Based on Greening (1976) Models

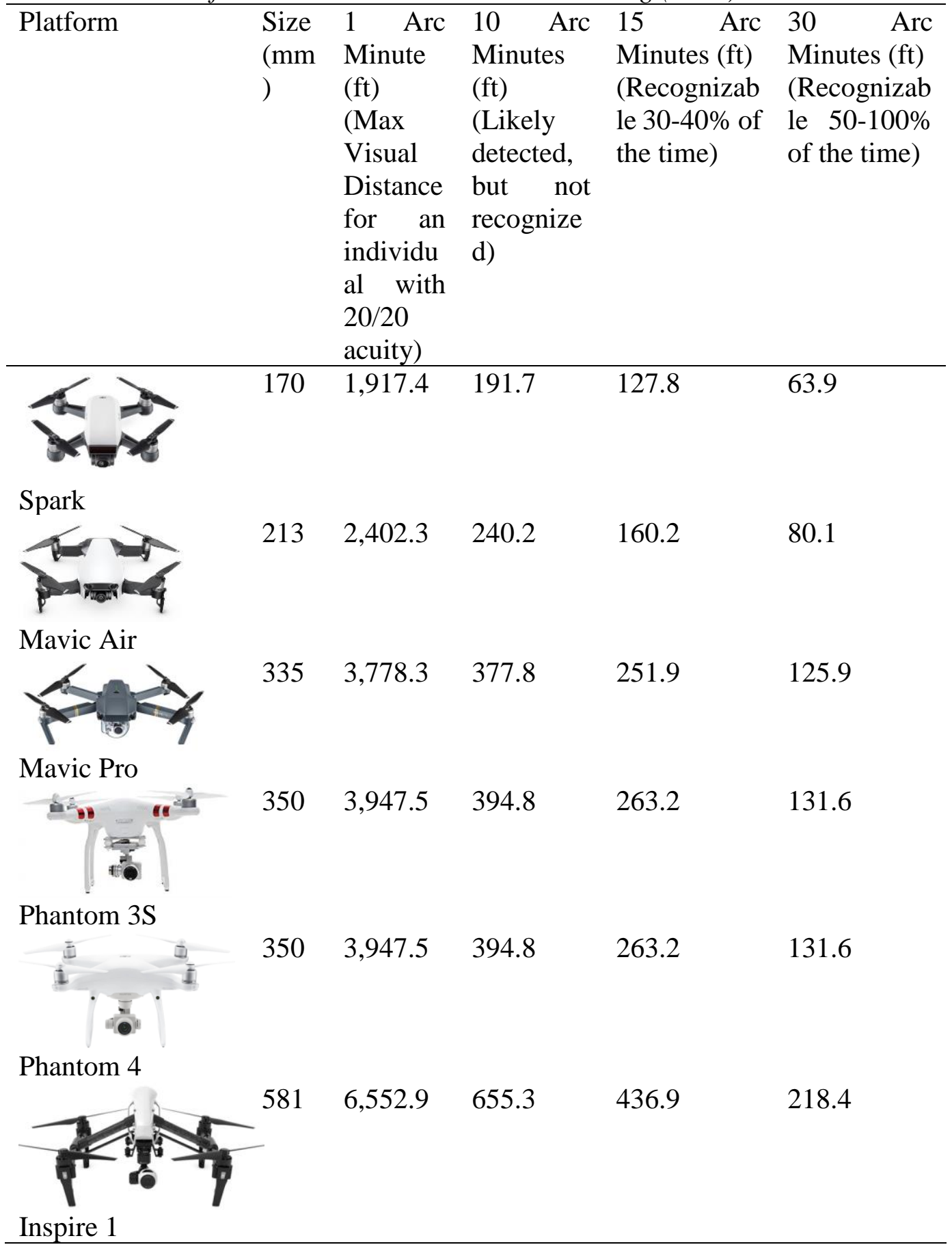




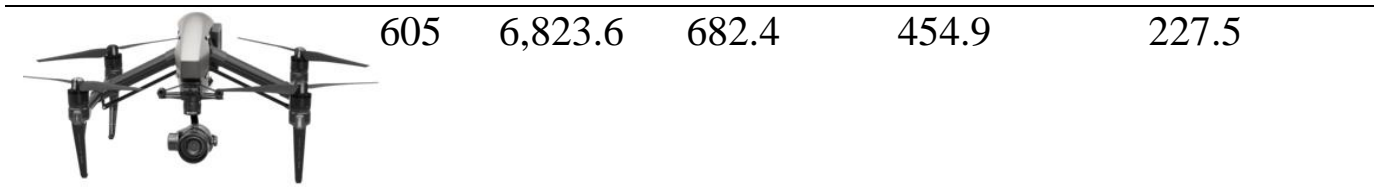

Inspire 2

Note: Size calculations performed without regard to props. Images are not to scale. Adapted from "Visual Detection of Small Unmanned Aircraft: Modeling the Limits of Human Pilots," by G. S. Woo, 2017, https://commons.erau.edu/edt/350, p. 32. Used with permission.

\section{Assumptions \& Limitations}

- The AeroScope is only equipped to detect sUAS manufactured by the DJI company.

- Distance measurements between the sUAS operator location and unmanned aircraft can only be carried out if both the unmanned aircraft and remote controller are situated within unobstructed line of sight of the AeroScope RF sensors. Additionally, the sUAS must have a fixed GPS signal for its own position

- Visibility modeling was performed using "best-case" scenario criteria, which included presumptions of high object contrast, favorable atmospheric visibility, adequate luminance, unobstructed line of sight, and 20/20 observer visual acuity.

- The lack of collected data points may not necessarily be inferential or represent operator flight behavior in other geographic areas.

\section{Findings \& Discussion}

\section{Sample Demographics}

Researchers deployed the AeroScope from July 22, 2018 to August 21, 2018 , in proximity to a regional airport in the southeastern United States. During the sampling period, the device collected 32,426 sUAS data points from 1,013 separate flights from among a population of 247 DJI-manufactured sUAS platforms. Only $10.9 \%(n=110)$ of the total flights contained data that allowed the authors to calculate distance information between the aerial vehicle and operator location. The distribution of detected platforms is contained in Figure 1. 


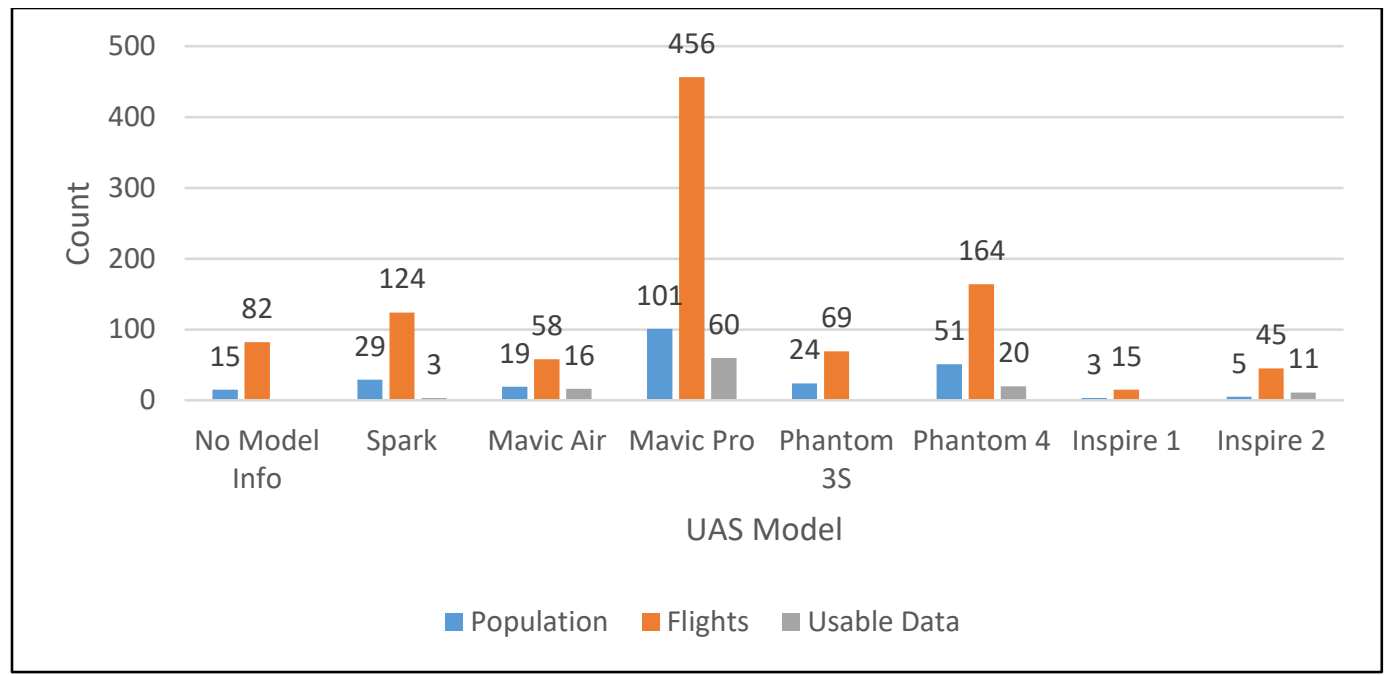

Figure 1. Distribution of detected sUAS by model. Population identifies the number of unique platforms. Flights are the total number of separate, continuous data detections. Usable data includes only those flights which included location information for both the DJI controller and aerial vehicle.

\section{UAS/Operator Lateral \& Vertical Offset Distance}

Lateral distance between the operator and aerial vehicle ranged from a minimum of 0 feet to a maximum of 7,596.5 feet. Aerial vehicle altitude ranged from 7.1 feet to 482.2 feet. Results are plotted in Figure 2. Summary statistics for distance, altitude, and slant range are presented in Table 3. Figure 4 shows the distribution of flights in 1/10 SM increments. 


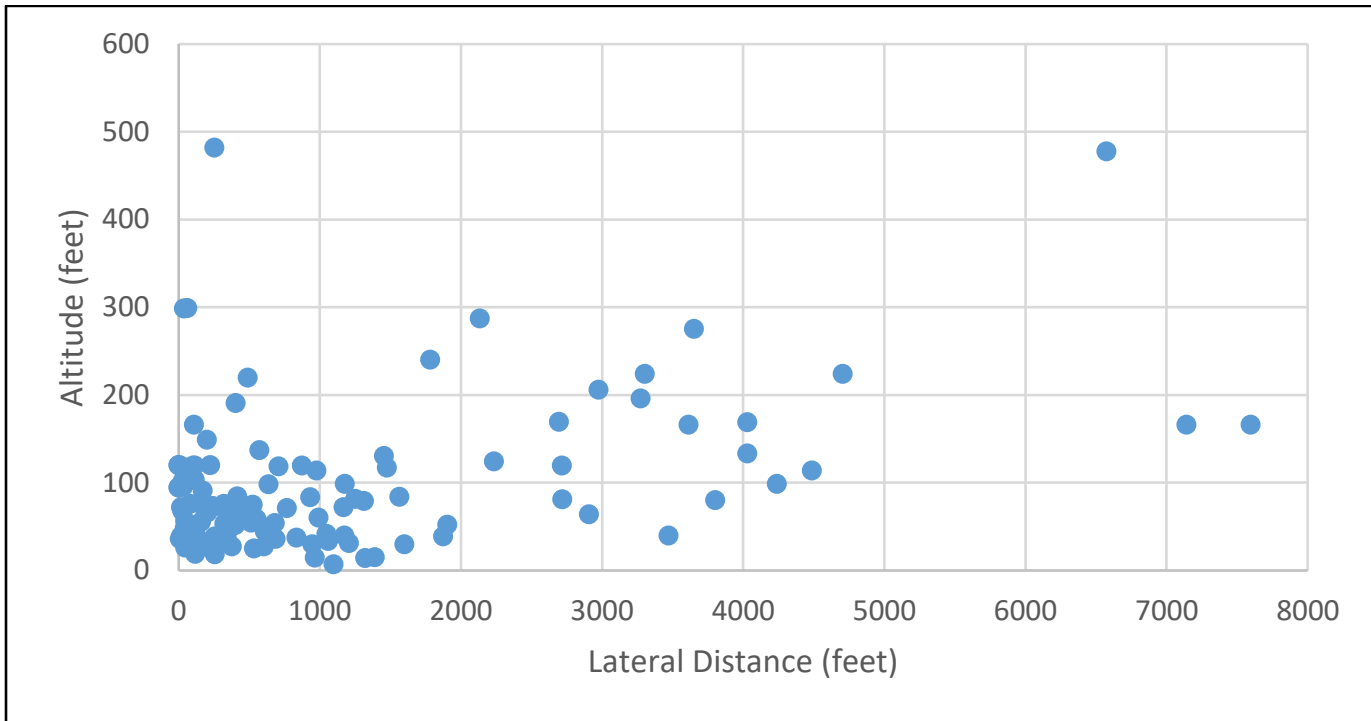

Figure 2. Distance and altitude of aerial vehicle relative to sUAS operator position plotted in feet.

Table 3

Summary Statistics of UAS Operator Offset Distance from Aerial Vehicle

\begin{tabular}{llll}
\hline & Distance & Altitude & Slant Range \\
\hline Min & 6.9 & 7.1 & 36.6 \\
Max & $7,596.5$ & 482.2 & $7,598.3$ \\
$\mu$ & $1,175.2$ & 98.8 & $1,236.7$ \\
$M$ & 523.1 & 74.8 & 536.2 \\
$\sigma$ & 1562.3 & 84.0 & $1,559.4$ \\
\hline
\end{tabular}




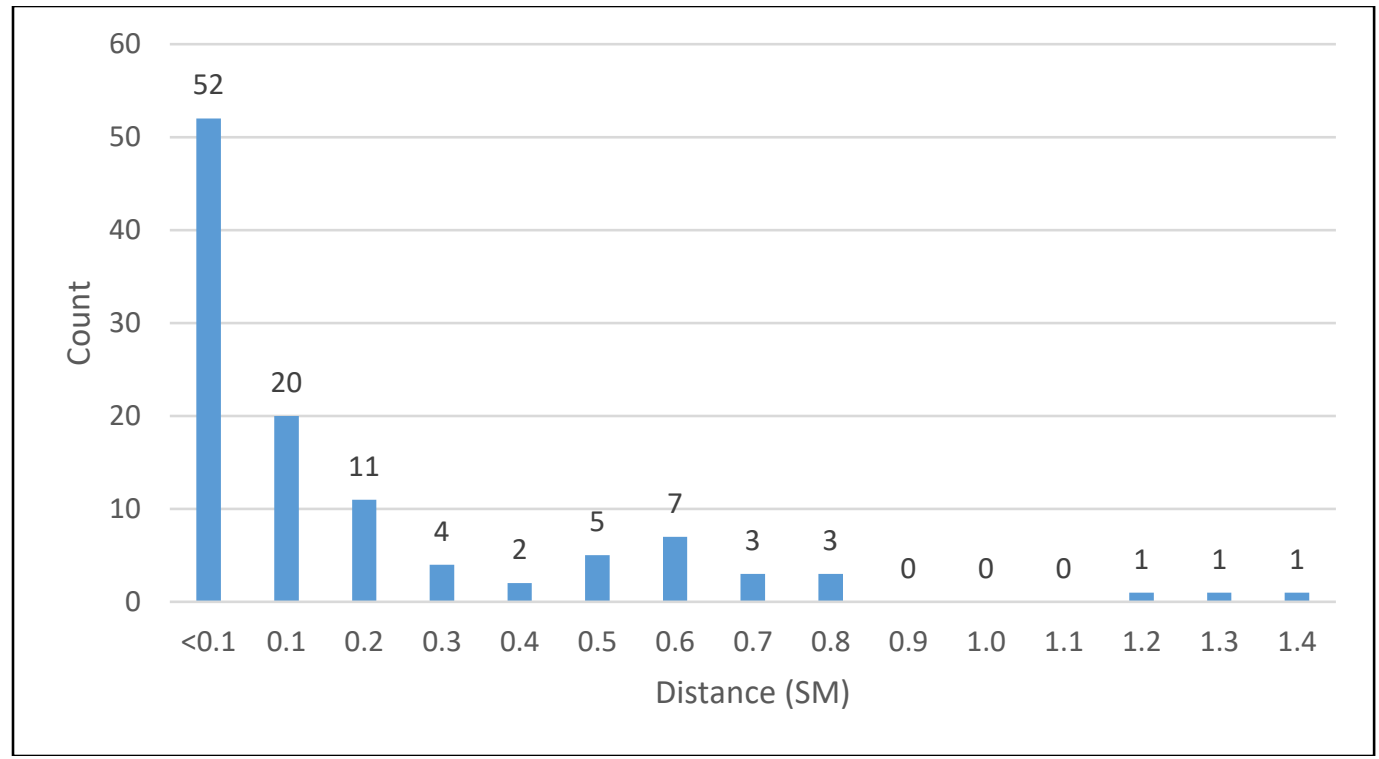

Figure 4. Histogram of detected sUAS max slant range in 1/10 SM bins.

The data showed variability, based on the sUAS model flown, with the smaller-sized aerial vehicles such as the MavicAir, MavicPro, and Spark being flown much closer to the operator than larger platforms. Summary statistics are presented in Table 4. Results are plotted in Figure 5.

Table 4

Summary Statistics of UAS Operator Slant Range Offset Distance from Aerial Vehicle by Model (ft)

\begin{tabular}{llllll}
\hline & Spark & MavicAir & MavicPro & P4 Series & Inspire 2 \\
\hline Min & 254.1 & 36.6 & 52.8 & 109.6 & 120 \\
$\operatorname{Max}$ & $1,207.90$ & $3,257.80$ & $4,241.00$ & $7,598.30$ & $6,591.50$ \\
$\mu$ & 638.5 & 594.4 & 701.1 & $2,584.20$ & $2,805.80$ \\
$M$ & 453.6 & 159.5 & 389.5 & $2,426.90$ & $3,278.90$ \\
$\sigma$ & 410.8 & 864.5 & 854.6 & $2,072.80$ & $1,833.70$ \\
\hline
\end{tabular}




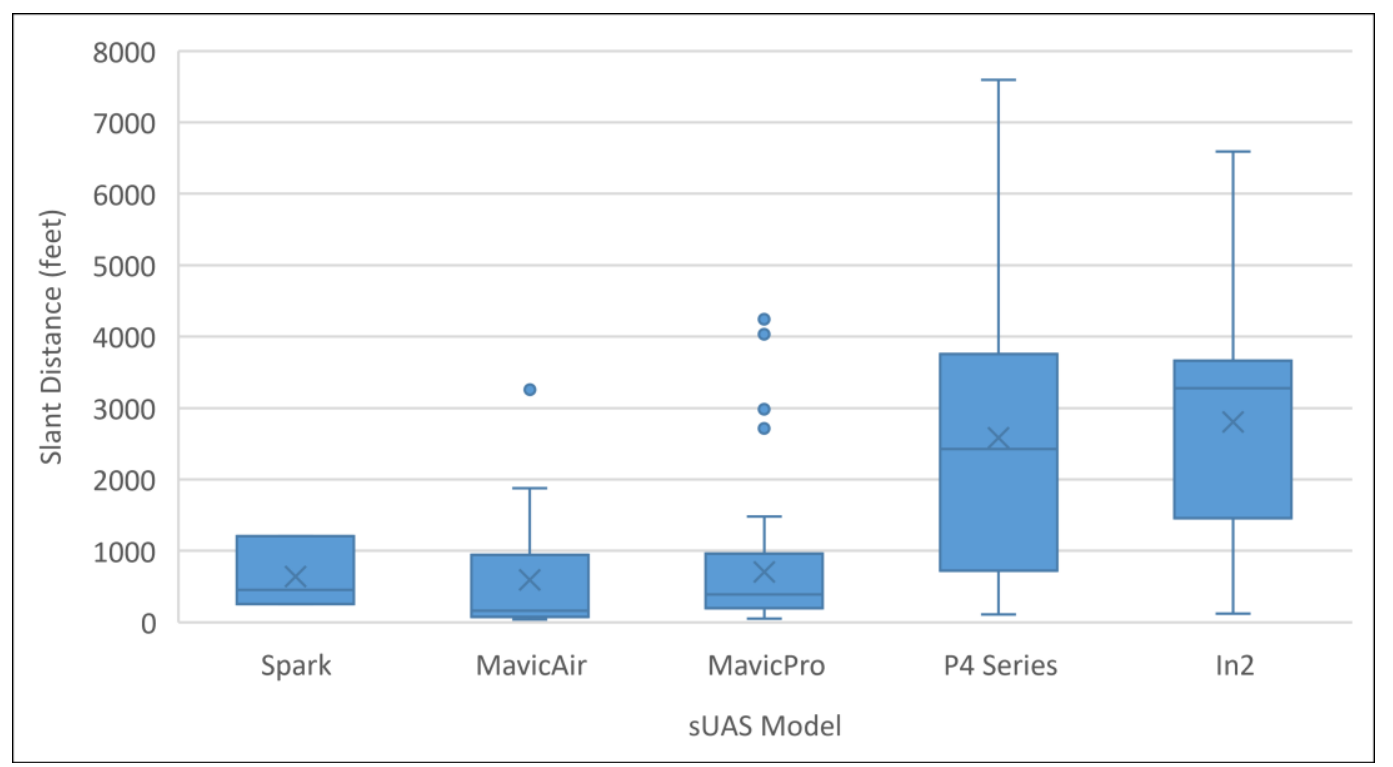

Figure 5. Box \& Whisker plot of detected sUAS Max Slant Flight Distances by platform type plotted in feet.

\section{Visibility Modeling}

Detected slant range distances for each sUAS model were compared against Greening's (1976) visual modeling. Results by model are presented in Figure 6; and, results by visibility category are presented in Figure 7. At least $5.5 \%$ of UAS flights $(n=6)$ were assessed as unlikely to be seen, with a calculated visibility of less than 1 arc-minute. Fifty-eight sUAS flights (52.7\%) met at least minimum requirements to be seen, with visibility of at least 1 arc-minute. Fourteen UAS flights $(12.7 \%)$ were likely to be visually detected but not necessarily recognized, with a visibility of at least 10 arc-minutes. Thirteen UAS flights $(11.8 \%)$ has a visibility of at least 15 arc-minutes and were likely to be recognizable $30-40 \%$ of the time. Sixteen UAS flights $(14.5 \%)$ were assessed to be recognizable greater than $50 \%$ of the time, with a visibility of at least 30 or more arc-minutes. It is important to note that visual angles greater than 10 arc minutes are not terribly relevant to this study, as the operator does not necessarily require recognition of the aerial vehicle, so long as he or she is aware of its relative position. Presumably, the operator knows the speck in the sky where their sUAS is expected is likely to be their drone. 


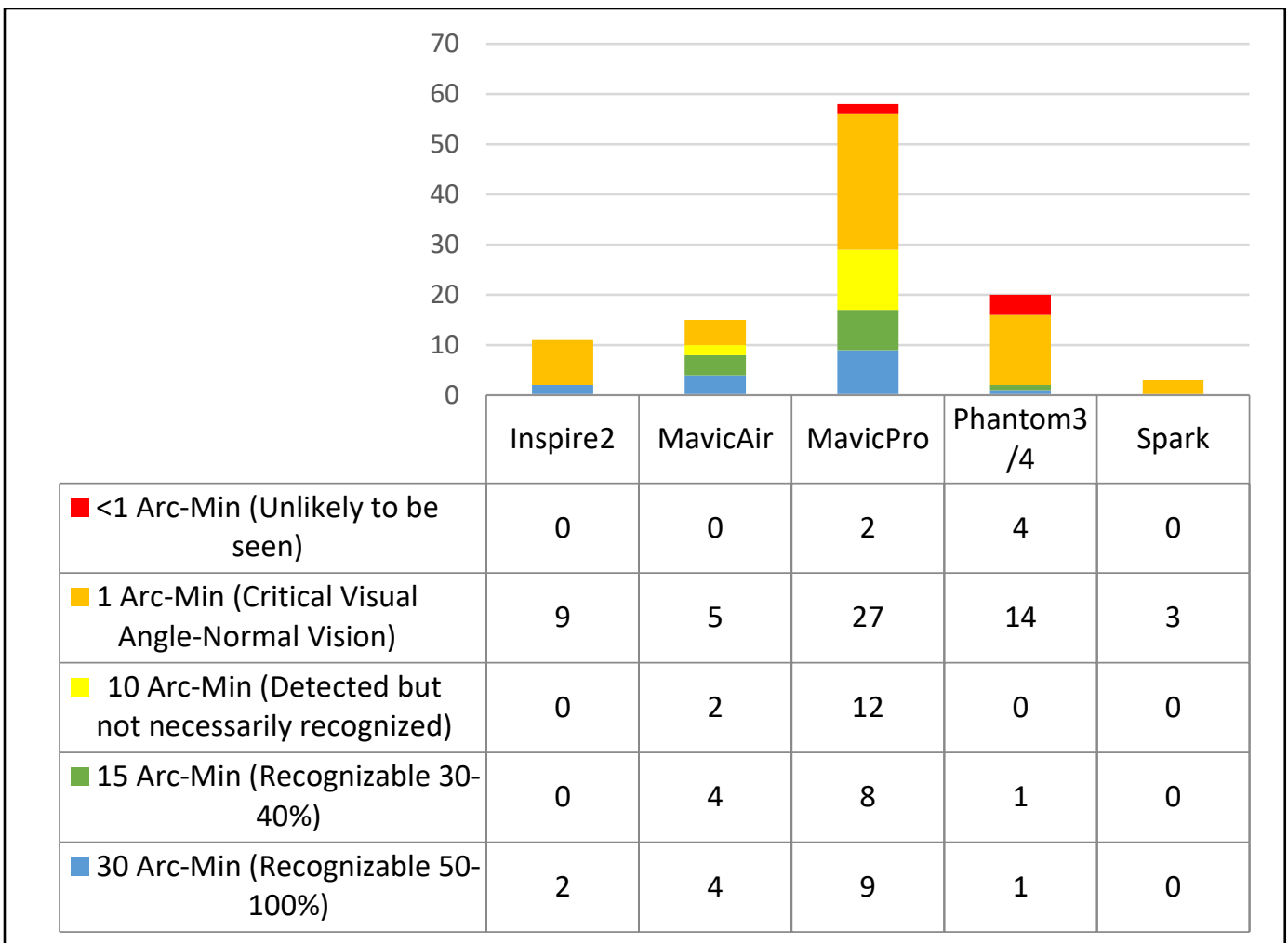

Figure 6. Detected sUAS model visibility based on Greening (1976) visibility modeling.

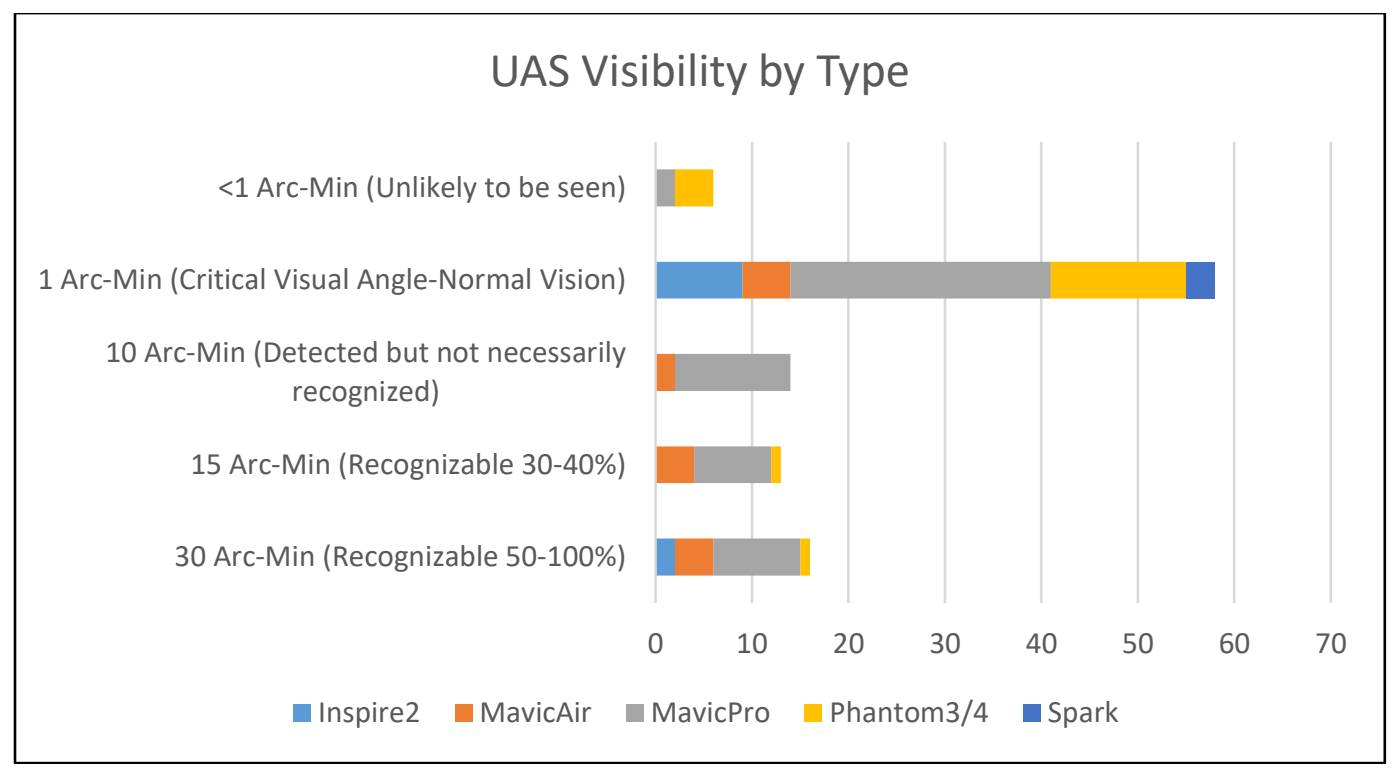

Figure 7. Detected sUAS visibility based on Greening (1976) visibility categories. 
Figure 8 presents a composite image of lateral and vertical sUAS detections, based on Greening's (1976) detectability model. Note how UAS visibility diminishes to less than 10 arc-minutes at distances greater than approximately 400 feet. Furthermore, all but the largest sUAS platforms are unlikely to be seen at distances greater than 4,000 feet.

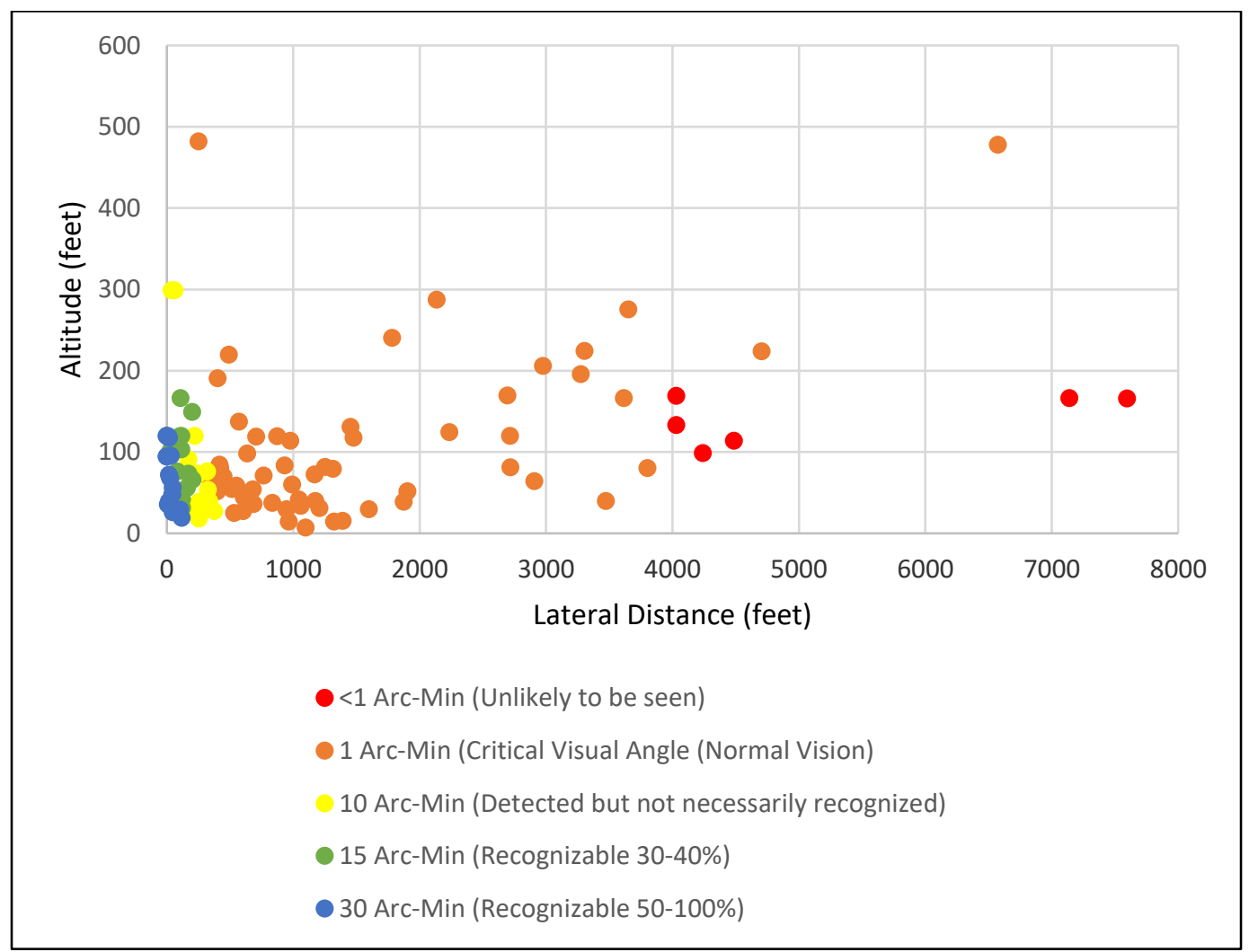

Figure 8. Model of Greening (1976) visibility based on UAS distance and altitude of aerial vehicle relative to operator position, plotted in feet.

\section{Case Studies}

The authors assessed the three data points detected furthest from their associated operators. Figure 9 depicts data point \#53, in which an operator flew his Inspire 2 nearly one and a quarter mile away, across a public golf course. The authors noted several single-story residences were aligned between the operator and unmanned aerial vehicle. . The authors did not assess if these buildings created a visual obstruction for the operator. An assessment of the operator's telemetry suggests that the AeroScope did not collect the full route of flight, likely due to 
obstructions to the sensor's line of sight. Visibility modeling suggests that barring any obstructions, the aerial vehicle may still have been visible to the operator.

Figure 10 depicts data point \#552 and 553, a DJI Phantom 4 flown nearly 1.5 SM from the operator. It is notable that the operator is positioned approximately one block from the shoreline, flying his unmanned aircraft over water. The authors noted that the operator's line of sight to the aerial vehicle is likely obstructed by a two-story structure located proximate to the beach. Visibility modeling indicates the aerial vehicle was unlikely to be seen by the operator

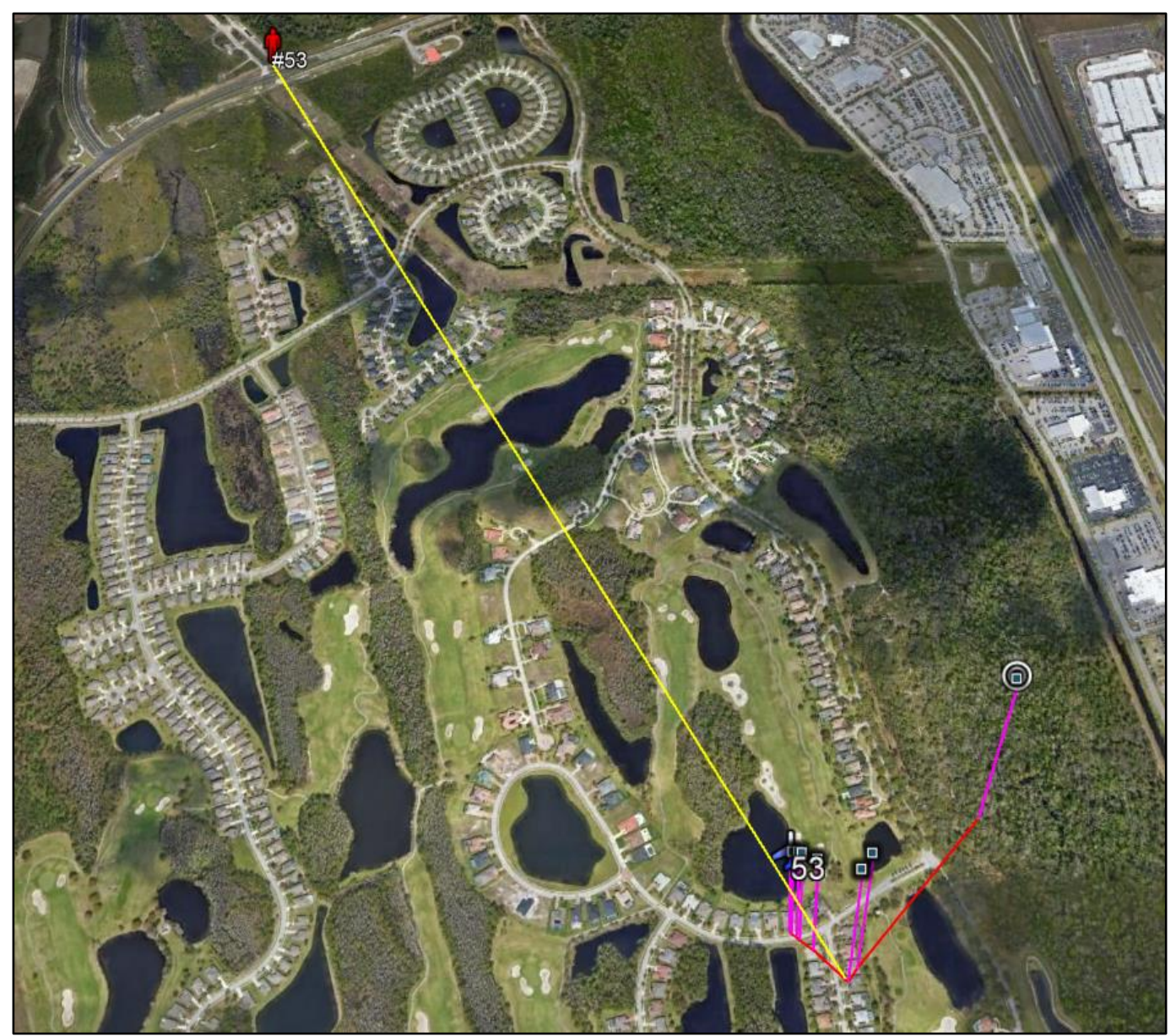

Figure 9. Overhead depiction of relative position between operator and aerial vehicle (Inspire 2), Data point \#53. Distance = 6,592 ft (1.24 SM). 


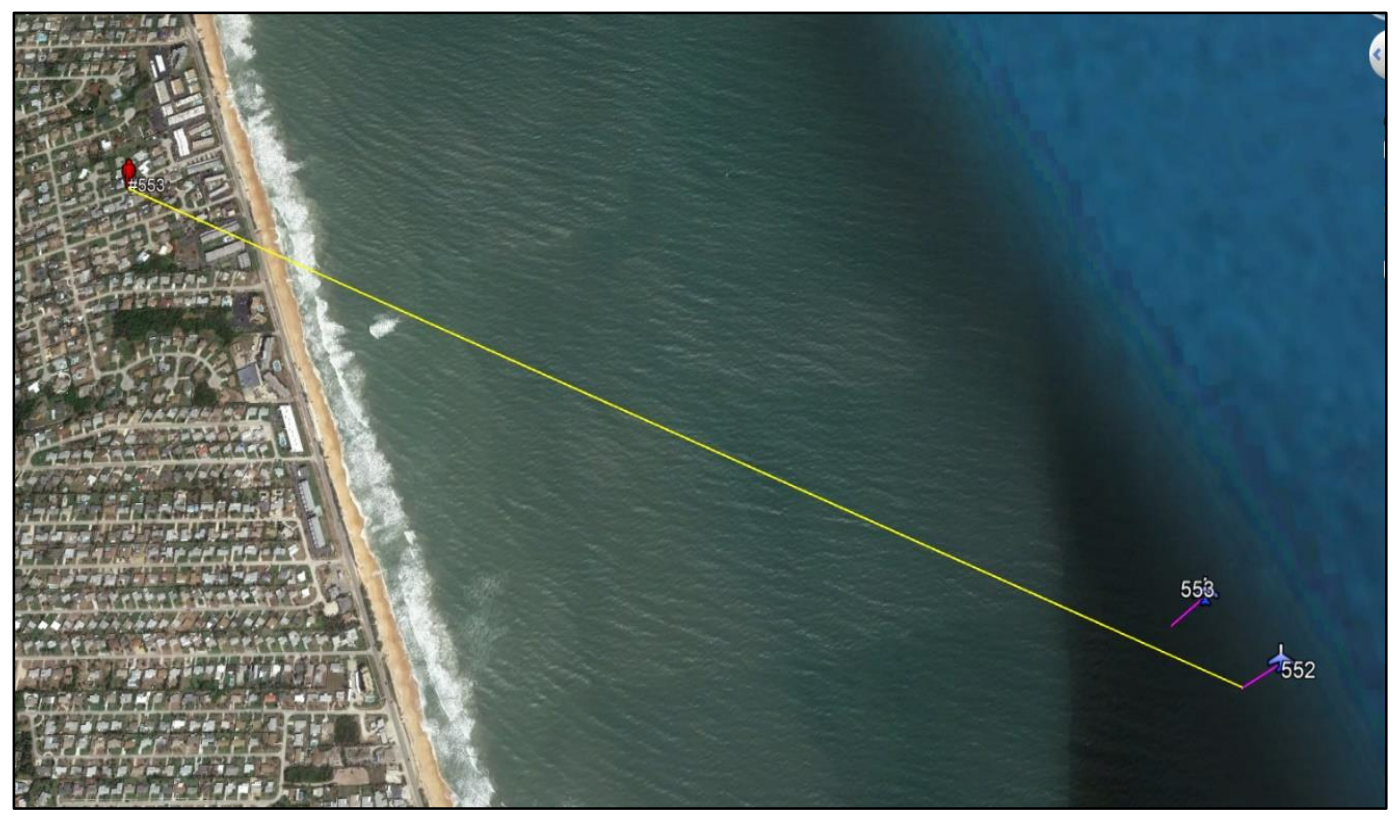

Figure 10. Overhead depiction of relative position between operator and aerial vehicle, Data points \#552 \& 553 (Phantom 4). Distance = 7,598 ft (1.44 SM) \& $7,142 \mathrm{ft}(1.35 \mathrm{SM})$, respectively.

\section{Additional Observations}

The authors made an additional, ancillary observation regarding sUAS operator orientation relative to their unmanned aircraft. The preponderance of unmanned aircraft $(n=67,60.1 \%)$ were flown at visual inclinations of less than 10-degrees from average human eye level of 5.75 feet (see Figure 11). This observation generally indicates that operators tend to fly further lateral distances than vertical distances. In a study of sUAS visual observers, Vance et al. (2017) found that participants who viewed aircraft/sUAS intercepts at higher angles of inclination encountered strong perceptual illusions that adversely impacted their ability to accurately judge vertical separation. The findings of this study suggest that the majority of UAS operators are unlikely to encounter this visual illusion. 


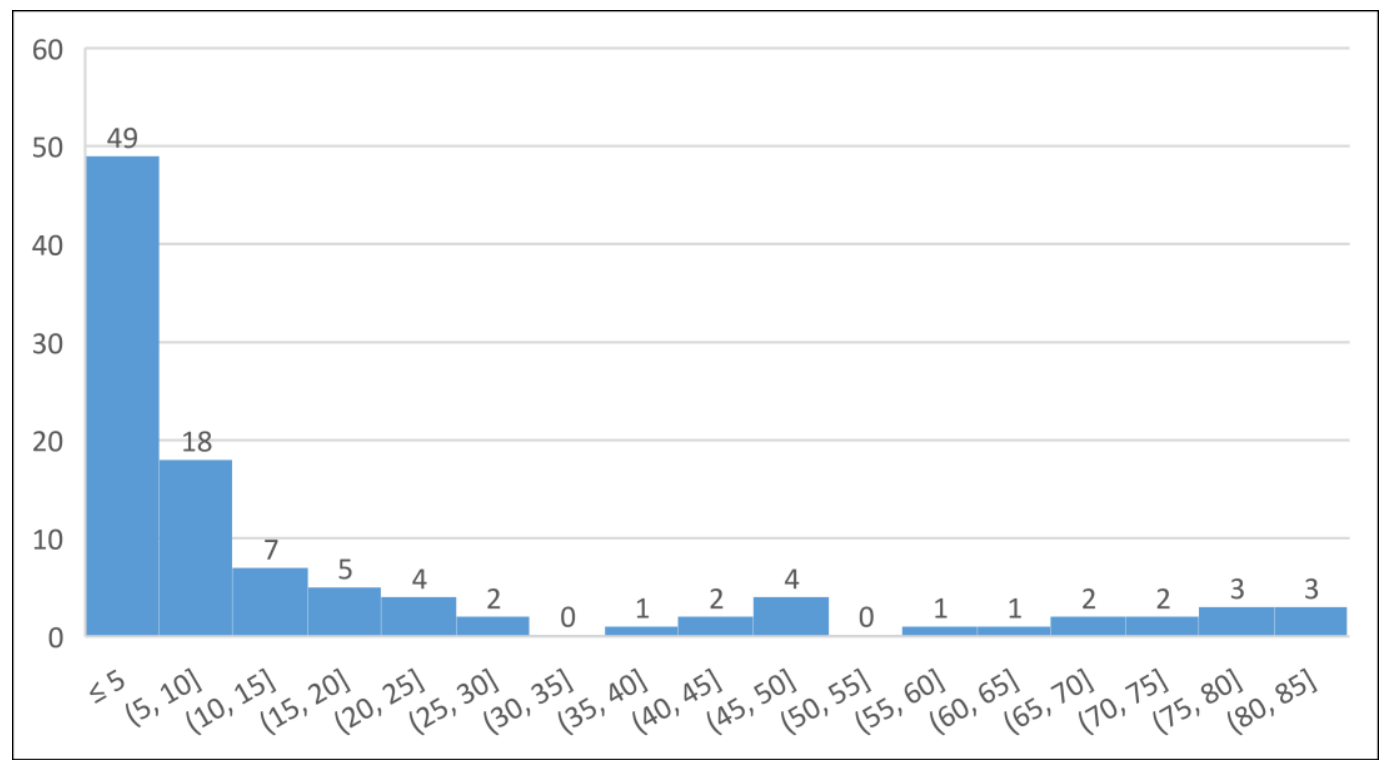

Figure 11. Histogram of operator viewing angle, based on distance and altitude relative to sUAS operator. Note: Based on average operator height of $5.75 \mathrm{ft}$.

\section{Conclusions}

\section{How far away do sUAS operators typically fly their unmanned aircraft?}

Collected data suggests that there is variability between operator flight distances, based on UAS model. Typically, smaller-sized models were flown at considerably closer distances than larger-sized models. For the smaller MavicAir, MavicPro, and Spark models, 75\% of flights occurred within 1100 feet of the operator, while for the larger Phantom and Inspire models, $75 \%$ of flights occurred within 3900 feet of the operator.

\section{What proportion of sampled flights were conducted Beyond Visual Line of Sight (BVLOS)?}

Analysis of collected data suggests that a majority of sUAS operators fly their craft within calculated limits for visual line of sight. When modeled using Greening (1976) methodology, nearly $94.5 \%$ of detected platforms were determined to be at least minimally visible, with a visual arc of at least 1 arc-minute or better. Six flights detected flights $(n=5.5 \%)$ were assessed to have been conducted beyond visual line of sight. While the data suggests the majority of sUAS operators are flying their platforms within visual line of sight, the number of detected BVLOS flights exceeded author expectations and warrant additional research. With the ever-increasing number of sUAS operations in the NAS, the risk 
of $5 \%$ of operations being conducted beyond the most conservative limits for line of sight represents a risk that must be mitigated.

The complexity and variability of operational environments make it difficult to identify a priori the maximum distance at which an aerial vehicle can be maintained within line of sight. While the theoretical value of 1 arc-minute is a useful place to start, field experiments show a great deal of variability in actual detection distances, both within and between subjects (Crognale, 2009). For example, based on the 1 arc minute criteria, the ScanEagle is theoretically detectable at $10.667 \mathrm{~km}(\sim 34,997 \mathrm{ft})$. However, in experimental settings, the mean distance at which visual contact was lost when the vehicle was flying away from the operator was $1.276 \mathrm{~km}(\sim 4,186 \mathrm{ft})$ (Crognale, 2009). The mean distance at which the ScanEagle was acquired when flying toward the operator was even lower, at $.898 \mathrm{~km}(2,946 \mathrm{ft})$ (Crognale, 2009).

\section{Recommendations}

Current separation between manned and unmanned aircraft is predicated upon airspace segregation and the sUAS operator's ability to detect and avoid manned aircraft through visual scanning. Ideally, sUAS operators would always be able to see their own aircraft. However, in more than 5\% of cases, operators could not see their own aerial vehicle, and therefore could not avoid nearby aircraft. Displaying nearby aircraft position and altitude on sUAS operator displays would enhance situational awareness for all sUAS operators, but in particular for those who have lost visual contact with their aerial vehicles.

\section{Future Research}

As demonstrated by the difference between the theoretical detection threshold and the actual thresholds in Crognale (2009), thresholds for visual contact may be much lower in empirical settings than in theory. Further research on fieldtested thresholds for visual detection would aid researchers in determining the risk posed by UAS operating at or beyond the threshold for visual detection. Models exist for estimating detection thresholds for manned aircraft, but these should be updated to accommodate sUAS, and validated empirically.

In future iterations of the study, the authors plan to include a diverse selection of sample locations. Additionally, the authors plan to incorporate 3-D visibility shed analysis to determine the effect of localized obstructions on UAS operator visual line of sight. 


\section{References}

Crognale, M. A. (2009). UAS ground observer performance: Field measurements (Report: DOT/FAA/AR-10/1). Washington, D.C.: Federal Aviation Administration.

Dolgov, I. (2016). Moving towards unmanned aircraft systems integration into the national airspace system: Evaluating visual observer's imminent collision anticipation during day, dusk, and night sUAS operations. International Journal of Aviation Sciences, 1(1). Retrieved from https://www.ijas.us/ index.php/issues/current-issues/issue-i-volume-1/articles/moving-towardsunmanned-aircraft-systems-integration-into-the-national-airspace-systemevaluating-visual-observers-imminent-collision-anticipation-during-daydusk-and-night-suas-operations-igor-dolgov

FAA Modernization \& Reform Act of 2012, Pub. L. 112-95, 126 Stat. 11, codified as amended at 49 U.S.C. 40101.

Federal Aviation Administration. (n.d.). 14 CFR Part 107 waiver safety explanation guidelines. Retrieved From https://www.faa.gov/uas/ commercial_operators/part_107_waivers/waiver_safety_explanation_guid elines/media/WSEG_operational_risks_mitigations.pdf

Federal Aviation Administration. (2016). Operation and certification of small unmanned aircraft Systems (RIN 2120-AJ60). Washington, D.C.: Author. Retrieved from https://www.faa.gov/uas/media/RIN_2120AJ60_Clean_Signed.pdf

Federal Aviation Administration. (2018). Part 107 waivers issued [database]. Retrieved from https://www.faa.gov/uas/commercial_operators/ part_107_waivers/waivers_issued/

Greening, C. P. (1976). Mathematical modeling of air-to-ground target acquisition. Human Factors, 18(2), 111-148.

Howett, G. L. (1983). Size of letters required for visibility as a function of viewing distance and observer visual acuity (National Bureau of Standards Technical Note 135 1180). Washington, DC: Government Printing Office. Retrieved from https://www.gpo.gov/fdsys/pkg/GOVPUB-C13ff8dc22d75e66f29ebdb2bb 2085ee683/pdf/GOVPUB-C13ff8dc22d75e66f29ebdb2bb2085ee683.pdf

Loffi, J. M., Wallace, R. J., Jacob, J. D., \& Dunlap, J. C. (2016). Seeing the threat: Pilot visual detection of small unmanned aircraft systems in visual meteorological conditions. International Journal of Aviation, Aeronautics, and Aerospace, 3(3). Retrieved from https://commons.erau.edu/ijaaa/vol3/ iss $3 / 13 /$

National Transportation Safety Board. (2017). Incident report \#DCA17IA202A. Washington, D.C.: Author. Retrieved from https://app.ntsb.gov 
/pdfgenerator/ReportGeneratorFile.ashx?EventID=20170922X54600\&AK ey $=1 \&$ RType $=$ HTML $\&$ IType $=$ IA

PrecisionHawk. (2018). Why 99\% of BVLOS Part 107 waivers are rejected. Retrieved from https://www.precisionhawk.com/media/topic/why-99-ofbvlos-part-107-waivers-are-rejected/

Small Unmanned Aircraft Systems, 14 C.F.R. § 107 (2016).

Terwilliger, B.A. (2012). The effect of visual interaction methods on simulated unmanned aircraft operator situational awareness (paper no. 12435). In Volume 2012: Proceedings of the 2012 Interservice/Industry Training, Simulation, and Education Conference (I/ITSEC). Arlington, VA: National Training and Simulation Association. Retrieved from https://commons.erau.edu/cgi/viewcontent.cgi? article=1553\&context=pub lication

Vance, S. M., Wallace, R. J., Loffi, J. M., Jacob, J. D., Dunlap, J. C. \& Mitchell, T. A. (2017). Detecting and assessing collision potential of aircraft and small unmanned aircraft systems (sUAS) by visual observers. International Journal of Aviation, Aeronautics, and Aerospace, 4(4). Retrieved from http://commons.erau.edu/ijaaa/vol4/iss4/4

Williams, K. W., \& Gildea, K. M. (2014). A review of research related to unmanned aircraft system visual observers (Report: DOT/FAA/AM-14/9). Washington, D.C.: Federal Aviation Administration. Retrieved from https://apps.dtic.mil/dtic/tr/fulltext/u2/1050266.pdf

Woo, G. S. (2017). Visual detection of small unmanned aircraft: Modeling the limits of human pilots (Doctoral Dissertation). Retrieved from https://commons.erau.edu/edt/350 